\title{
Le Tribunal fédéral fait dans le «sur-mesure»
}

\author{
Hans Georg Kopp \\ Dr med., spécialiste en psychiatrie et psychothérapie, FMH, Centre d'expertises, Clinique de réadaptation de Bellikon
}

Le Tribunal fédéral a changé fondamentalement sa pratique pour l'évaluation des troubles somatoformes. La capacité de travail réellement exigible des personnes concernées devra désormais être évaluée dans le cadre d'une nouvelle procédure d'instruction structurée à l'aide d'«indicateurs standards». Ce changement de jurisprudence valorise l'importance de l'expertise psychiatrique différenciée.

\section{Contexte}

Les patients souffrant de douleurs persistantes non explicables par des causes organiques posent un problème à la médecine. Ils se situent comme dans une zone grise entre le corps et l'esprit. Sur le plan conceptuel, la classification psychiatrique sous le terme de «trouble somatoforme» ne couvre en particulier pas de manière suffisante le large spectre des troubles douloureux chroniques. Leur évaluation en termes de diminution effective de la capacité de travail était, et demeure, difficile.

Face à la forte progression des rentes attribuées dans ce contexte dès la fin des années 90 sur la base du diagnostic de troubles somatoformes douloureux et persistants, le Tribunal fédéral a réagi en 2004 en définissant les critères dits de Foerster. Cette liste de critères destinée aux praticiens du droit a alors été appliquée de manière très schématique. Ce schématisme des assurances sociales et des tribunaux a suscité de nombreuses critiques au cours des années. Dans les milieux psychiatriques, le sentiment d'être incompris dans son argumentation professionnelle était très répandu.

Dans son arrêt du 3 juin 2015, le Tribunal fédéral a désormais modifié sa pratique et défini une procédure d'instruction structurée à l'aide d'indicateurs (et non de critères «à cocher») permettant d'orienter le travail d'évaluation ainsi que l'argumentation. Ces résultats doivent ensuite être présentés dans leur contexte et pondérés de façon convaincante dans le cadre de l'évaluation de l'expert. L'arrêt du Tribunal fédéral montre que le tribunal s'est longuement penché de manière différenciée sur la littérature scientifique existante et sur les avis d'experts concernant les troubles somato- formes. Certains représentants du Tribunal fédéral ont également assisté avec intérêt aux conférences organisées sur le sujet ces dernières années. L'expertise fondamentale de Peter Henningsen (Université Technique de Munich) a largement contribué au changement de jurisprudence (à consulter sur le site du Cabinet d'avocats «indemnis»).

D'un point de vue psychiatrique, la nouvelle liste d'indices définis par le Tribunal fédéral répond de manière adéquate à la problématique de l'affection psychosomatique et intègre les aspects pertinents de ces troubles, notamment le degré de gravité, ainsi que l'impact de la structure de personnalité sous-jacente et les ressources personnelles et sociales qui en découlent. Elle amène également à évaluer le contexte

L'objectif poursuivi est une plus grande fiabilité, objectivité et transparence dans le processus d'évaluation de l'expertise.

social et son influence sur les symptômes de la douleur. Une attention particulière est accordée à l'appréciation minutieuse de la cohérence de l'expertise.

Cette nouvelle pratique ne change donc pratiquement rien à la procédure d'évaluation, si on la compare aux Lignes directrices de qualité pour les expertises psychiatriques formelles de la SSPP (2012).

\section{Aspect pratique de l'expertise: des problèmes en suspens demeurent}

Malgré tous les éléments positifs de cet arrêt du Tribunal fédéral, divers aspects de ces expertises demeurent difficiles. L'auteur se base ici sur sa propre expérience pratique dans l'évaluation interdisciplinaire de tels 
troubles dans le cadre du Centre d'expertises de la Clinique de réadaptation de Bellikon.

Même si le Tribunal fédéral définit les principales problématiques qu'il convient d'évaluer et d'apprécier à l'aide de sa grille d'examen structurée et normative, la collecte d'informations pertinentes et de résultats justifiables pour l'expertise demeure dans la pratique un défi majeur. Voici un bref rappel des points délicats:

Phase diagnostique: les critères de la CIM-10 pour définir les troubles somatoformes ainsi que les troubles somatoformes douloureux persistants sont au plan conceptuel parfois problématiques ou flous, et peu pertinents en regard du degré de gravité; l'applicabilité des critères selon le DSM-5 devra d'abord faire ses preuves dans la pratique d'évaluation. Par ailleurs, l'éventail des patients souffrant de douleurs chroniques est vaste et divers, la majorité d'entre eux a subi des processus d'apprentissage dysfonctionnels et importants pour le comportement, et seule une minorité souffre d'un syndrome somatoforme au sens strict du terme. Toutefois, chez les patients souffrant de douleurs dysfonctionnelles ne répondant pas aux critères de la CIM-10 ou du DSM-5, l'expérience montre qu'en règle générale, le degré de gravité fonctionnel requis selon les critères du Tribunal fédéral - n'est pas rempli. Les situations de patients souffrant de douleurs chroniques avec leur multitude de facteurs d'influence psychosociaux sont cependant très complexes - une complexité à laquelle des distinctions diagnostiques catégorielles apparemment simples ne sauraient répondre. La phase diagnostique comme critère décisif préalable en vue de l'appréciation juridique ultérieure des réflexions de l'expertise peut ainsi devenir une pierre d'achoppement.

L'évaluation de la personnalité est un processus exigeant. En règle générale, les affections psychosomatiques sont plus des anomalies structurelles du «moi» (au sens de traits de personnalité accentués) que de véritables troubles de la personnalité. Or, le contexte d'une expertise (parfois après des années de litige avec l'assurance) est souvent peu propice à un diagnostic nuancé de la personnalité. Il est au contraire souvent nécessaire de tirer des conclusions sur la structure de la personnalité à partir de l'interaction observée, de la biographie et de l'aménagement de la situation de vie, y compris de la gestion de la symptomatologie douloureuse.

Les conséquences fonctionnelles de l'affection en termes de capacité de travail peuvent être par ex. déduites de manière différenciée d'une procédure selon le MiniICF, même si cet instrument n'est pas encore validé pour le contexte des assurances médicales et ne présente pas une fiabilité suffisante selon les résultats de premières études. Des observations comportemen-

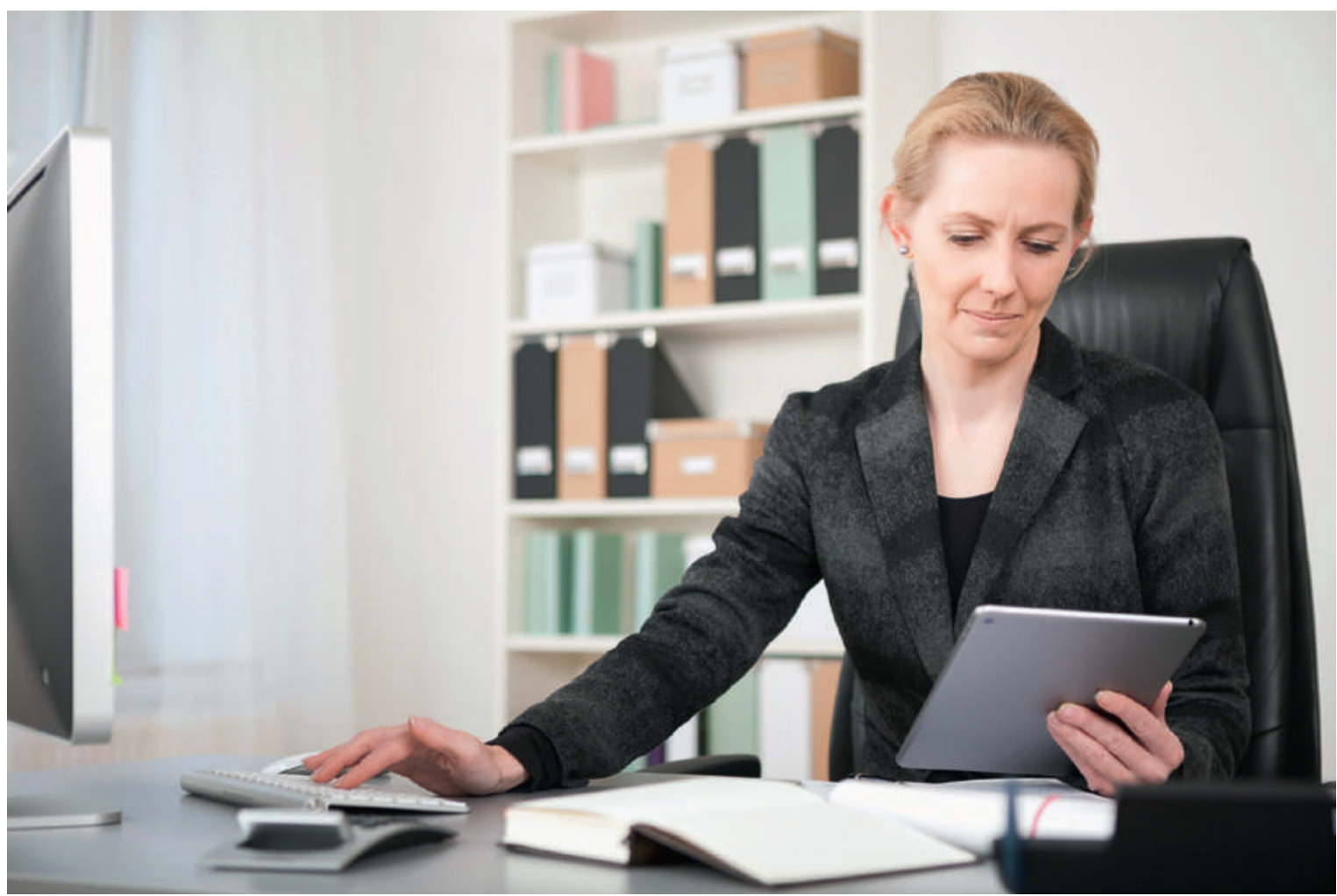

La charge de travail correspondante pour les experts est certes considérable, tout en restant dans le cadre déjà défini par les Lignes directrices de qualité pour expertises psychiatriques de la SSPP. 
tales effectuées dans le cadre d'une instruction interdisciplinaire à l'aide d'évaluations pratiques standardisées par des thérapeutes spécialement formés - comme c'est le cas à Bellikon - sont davantage aptes à fournir, en plus des informations verbales, des résultats objectifs pour évaluer le degré fonctionnel et la cohérence. Pour ce type d'affections, il est courant de supposer des schémas complexes d'incohérences. Parfois spécifiques au trouble, ces incohérences sont parfois aussi le résultat de processus d'apprentissage dysfonctionnels ou résultent d'une déformation volontaire. L'analyse de la cohérence intègre à juste titre davantage d'éléments et d'observations que ceux énumérés par le Tribunal fédéral sous la catégorie "Cohérence». Une vaste

\section{La phase diagnostique comme critère décisif préalable en vue de l'appréciation juridique ultérieure des réflexions de l'expertise peut ainsi devenir une pierre d'achoppement.}

expérience de l'appréciation de la cohérence peut être très utile, et une appréciation interdisciplinaire qui intègre, le cas échéant, la neuropsychologie et ses tests de validation des symptômes, soutient une expertise consensuelle et interdisciplinaire de la cohérence. Il faut toutefois éviter de tirer des conclusions générales à partir d'incohérences isolées. Un texte d'appréciation compréhensible, convaincant et largement étayé demeure l'élément central de ces expertises.

Dr Hans Georg Kopp

Centre d'expertises de

la Clinique de réadaptation

de Bellikon

CH-5454 Bellikon

Tél. 0564855439

Fax 0564855484

kopp[at]rehabellikon.ch particuliers, lorsque ces dernières sont élaborées de manière professionnelle. La charge de travail correspondante pour les experts est certes considérable, tout en restant dans le cadre déjà défini par les Lignes directrices de qualité pour expertises psychiatriques de la SSPP. On peut espérer que des expertises différenciées conduiront à une nouvelle différenciation dans l'appréciation de toutes les manifestations psychosomatiques. Toutefois, avec l'appréciation objective des argumentations d'expertise, les assurances et les tribunaux seront encore davantage sollicités.

Une exception pour les expertises des affections psychosomatiques et des troubles dits fonctionnels est finalement dépourvue de toute justification rationnelle, puisque presque tous les troubles psychiques ne présentent aucune cause organique; c'est pourquoi les expertises se fondent beaucoup sur les informations des proches dont elles doivent vérifier la plausibilité. Il est donc judicieux de procéder à un aménagement concret des directives pour les expertises en vue d'une évaluation uniforme de l'ensemble des affections psychiques et psychosomatiques, en mettant l'accent sur la déduction logique de conséquences pour la capacité de travail «exigible». L'objectif poursuivi est une plus grande fiabilité, objectivité et transparence dans le processus d'évaluation de l'expertise.

Un tel groupe de travail, composé de représentants des disciplines médicales somatiques compétentes et de la psychiatrie et placé sous la responsabilité de la SSPP, a commencé ses travaux dans ce sens. Les indications correspondantes dans l'arrêt du Tribunal fédéral font office de mandat officiel.

Remerciements

L'auteur remercie les Docteurs H. J. Mosimann et G. Ebner pour leurs précieuses suggestions. 